\title{
Comment about the Letter entitled "Scalar fields as dark matter in spiral galaxies"
}

\author{
Daniel Sudarsky \\ Instituto de Ciencias Nucleares \\ Universidad Nacional Autónoma de México \\ Apdo. Postal 70-543 México D.F. 04510, México
}

November 21, 2018

The objective of this brief comment is to point out several problems associated with the general framework underpining this paper and with the application in this work in particular. For one, it is easily proven that there are no non-singular asymptotically flat, static solutions to the coupled Einstein Scalar Field theory. Thus, the solution(s) presented in [1], ought to be singular, and therefore can not be considered as corresponding to a real physical galaxy, in whose center we expect to have either a black hole or just a region with high density ordinary matter. One could have hoped that one of the situations above would allow one to remove the singularity. Unfortunately this will not work with either of these two alternatives because, in the black hole case, the no hair theorems would rule out the static solution from the start, and in the ordinary matter case, the very stringent experimental bounds for the coupling of such a scalar field with ordinary matter would disallow the consideration of ordinary matter as the "source" of the field. Moreover if one takes the view that ordinary matter at the galactic center is responsible for the nontrivial configuration of the scalar field, one needs to explain why for example a similar phenomena doesn't happened, say, at the solar system scale.

On the other hand if we take a close look at the analysis of the motion of test particles in [1] we find a very serious flaw: in arriving at eq. 18 which is supposed to give the condition for circular orbits the authors just set $\dot{\rho}=0$ in the "energy conservation equation" without verifying that the point corresponds to a minimum of the effective potential. This would correspond to finding the turning points of the various non-circular orbits rather than the condition for circular orbits which is what is needed. Moreover, one can go directly to the final expression for the metric, eq.(21) of [1] , and see explicitly that there are in general no circular orbits as the effective potential for the motion on the equatorial plane is $V_{\text {eff }}(r)=\left(f_{0} / r_{0}\right)\left((r-a)^{2}+K^{2}+f_{0} r_{0} B^{2}\right)$ which has no minima other than $r=a$, a point where the coordinates are not longer valid. Thus there are no "rotation curves" associated with the spacetime described in this work. Finally even if there were such orbits in the spacetime associated with the scalar field configuration and they yielded 
the expression in eq. 20 for the velocity of rotation, one could not justify the procedure used by the authors to take into account the effects of the visible matter. Namely to

take the resulting velocity to be $v_{T}=\sqrt{V_{D M}^{2}+V_{L}^{2}}$ where $V_{L}$ represents somehow the "circular velocity of a star which would be associated with the luminous matter". There is absolutely no justification for this pythagorean addition of velocities since they are not orthogonal nor do they represent statistically uncorrelated averages.

We must therefore conclude that our colleagues have unfortunately been led astray by the enthusiasm in their mistaken belief of having found a solution to this most intriguing problem.

\section{Acknowledgments}

This work was in part supported by DGAPA-UNAM grant No IN121298 and by CONACyT grant 32272-E.

\section{References}

[1] T. Matos and S. Guzman. Class. and Quantum Grav. 17 L9, (2000). 